\title{
Las cadenas globales de valor de las manufacturas en México (2005-2015)
}

\author{
Flor Amparo Medina Chamorro* \\ Nicolás De la Peña Cárdenas ${ }^{* *}$
}

Fecha de recepción: 13 de septiembre de 2019

Fecha de aprobación: 2 de diciembre de 2019

Resumen: México es una de las economías más insertadas en cadenas globales de valor, especialmente con Estados Unidos en la industria automotriz. Este artículo tiene por objeto analizar la integración del sector manufacturero de México en las cadenas globales de valor (CGV), observando la profundidad de las actividades de transformación, los sectores más dinámicos y la importancia para el mercado laboral. Para ello se utilizan las bases de datos Trade in Value Added (TiVA) y Trade in eMployment (TiM) publicadas por la OCDE. Se encuentra que el sector de manufacturas mexicano está fuertemente integrado en CGV, en especial con Estados Unidos, pues abarca una elevada proporción de las exportaciones con valor agregado y de los empleos generados por exportaciones. No obstante, la integración del sector lo expone a dos desafíos principales: los cambios en la política comercial de Estados Unidos y la automatización.

Palabras clave: cadenas globales de valor, manufacturas, México, valor agregado, empleo.

Clasificación JEL: F1, F16, F4, F6, L6.

Cómo citar este artículo/ To reference this article / Comment citer cet article / Para citar este artigo:

Medina Chamorro, F., \& De La Peña Cárdenas, N. (2020). Las cadenas globales de valor de las manufacturas en México (2005-2015). Apuntes Del Cenes, 39(69). Págs. 13 - 38

https://doi.org/10.19053/01203053.v39.n69.2020.9998

\footnotetext{
* Economista, magíster en Dirección y Administración de Empresas, docente tiempo completo Facultad de Ciencias Sociales y Empresariales, Universidad Piloto de Colombia (Bogotá, Colombia). Correo electrónico: amparo-medina@unipiloto.edu.co Bhttp://orcid.org/0000-0002-1500-7278

** Profesional en Comercio Internacional, magíster en Asuntos Internacionales. Profesor de tiempo completo, Facultad de Ciencias Económicas y Sociales, Universidad de La Salle (Bogotá, Colombia). Correo electrónico: ndelapena@ unisalle.edu.co Bhttps://orcid.org/0000-0001-7223-9502
} 


\title{
Global Value Chains of Manufacturing in Mexico (2005-2015)
}

\begin{abstract}
Mexico is one of the most integrated economies in GVC, particularly with the United States in the automotive industry. The objective of this article is to analyze the integration of the manufacturing sector in $\mathrm{CGV}$, taking into account the deepening of transformation activities and the relationship with labor markets. In doing so, we use Trade in Value Added (TiVA) and Trade in eMployment (TiM) databases published by the OECD. We found that Mexico's manufacturing sector is strongly integrated in GCV, specially with the United States, covering a high proportion of added-value exports and jobs created by exports. Nonetheless, the integration exposes Mexican manufacturing sector to two main challenges: US trade policy changes and automation.
\end{abstract}

Keywords: global value chains, manufacturing, Mexico, value added, employment. 


\section{INTRODUCCIÓN}

El aumento de la integración económica que genera la globalización, ha traído consecuencias para la economía mexicana. En especial, la profundización de las cadenas globales de valor ha hecho que la producción sea más dependiente del comercio internacional, de modo que los cambios en la economía global tienen repercusiones para la economía nacional, las empresas y los trabajadores.

Las decisiones recientes del Gobierno de Estados Unidos referentes a la política comercial, entre ellas la renegociación del TLCAN, tiene implicaciones para México, por cuanto afectan las condiciones comerciales con Estados Unidos y Canadá, sus principales socios comerciales. Para el año 2018, el 83\% de las exportaciones mexicanas tuvieron como destino a sus vecinos del norte. No obstante, parte de ese valor comerciado está constituido por actividades de procesamiento, lo que quiere decir que el valor agregado de México es menor que lo reportado por las estadísticas brutas de exportación.
Esta investigación es de carácter exploratorio y busca responder la pregunta ¿cuál es la situación de la integración manufacturera de México en las cadenas globales de valor y la caracterización del empleo que resulta de este proceso? Para ello, se utilizan bases de datos elaboradas por la OCDE y la $\mathrm{OMC}$, tomando como referente seis indicadores de cadenas globales de valor. Además de la presente introducción, el artículo consta de los siguientes apartados. En el primero, marco teórico, se discuten las motivaciones para que los países se inserten en cadenas globales de valor, así como sus implicaciones. En el segundo se presentan las bases de datos utilizadas y la metodología. En el tercero, panorama comercial, se describen características generales del sector externo mexicano. En el cuarto, impacto sobre el comercio internacional, se muestra el nivel de integración de México con las cadenas globales de valor en la industria manufacturera. En el quinto, impacto sobre el empleo, se exponen algunas implicaciones de las cadenas globales de valor en el mercado de trabajo. Finalmente, se presentan las conclusiones. 


\section{MARCO TEÓRICO}

De acuerdo con los postulados de la nueva geografía económica desarrollada por (Krugman, 1979 - 1991), los patrones de comercio internacional se explican por las economías de escala y la reducción de costos de transporte. El interés de las compañías multinacionales por aumentar la rentabilidad conduce a la reducción de los costos de producción, haciendo uso de las economías de escala. La ubicación de las plantas de producción responde a cuestiones geográficas como los salarios y el conocimiento de los trabajadores, que se pueden explotar con mayor facilidad debido a la reducción de costos de transporte. Por otro lado, la reducción de los costos de comunicación facilita las transacciones y logra conectar con mayor facilidad oferta y demanda (Feenstra, 1998), así como la reducción de distorsiones asociadas a las asimetrías de información (Steinwender, 2018), lo que contribuye a facilitar la coordinación entre la casa matriz y sus diferentes unidades de producción en el mundo. Además, la liberalización global del comercio facilitada por la Organización Mundial del Comercio (OMC) y los acuerdos comerciales favorecieron el movimiento de mercancías a un menor costo (Aichele \& Heiland, 2018). En consecuencia, el interés de las compañías multinacionales, junto con mejoras en las tecnologías de información y la comunicación (TIC), así como la reducción en costos de transporte y en las barreras comerciales, han permitido la reconfiguración del comercio global.
De acuerdo con (Baldwin, 2016), existen tres transformaciones principales del comercio internacional. Hasta finales del siglo XIX, las fábricas producían cerca de los consumidores y se encargaban de la totalidad del proceso manufacturero, pero con la expansión global de la máquina de vapor y las consecuentes mejoras en transporte, la oferta y la demanda se separaron geográficamente, con productores en un punto y compradores en otro, lo que representa la primera transformación. La continua reducción de costos de transporte y comunicación separó geográficamente a los productores, cuya ubicación empezó a estar determinada por dotación factorial y costos salariales, constituyendo así la segunda transformación del comercio internacional. La última de estas transformaciones surgió al final del siglo XX como consecuencia del surgimiento de las TIC, las cuales permitieron mover libremente las ideas en diferentes puntos, fragmentando no solamente el proceso productivo sino los procesos más complejos de creación de valor, como el diseño. Cabe resaltar que esto representa una oportunidad para los países en desarrollo, por cuanto les permite utilizar a sus trabajadores en estas labores, lo que de otra manera sería imposible dada la distancia física que los separa de las centrales de las multinacionales. Esta tendencia se ha visto reforzada por el surgimiento de tecnologías de aprendizaje automático como la inteligencia artificial, que abren las puertas a una nueva competencia hacia la máxima reducción de costos, 
aumentan la productividad de los trabajadores con estos sistemas y logran separar la cadena de valor de los servicios (Baldwin, 2019).

La teoría de los costos de transacción (Coase, 1937) implica que una firma realiza actividades en la medida en que los costos de transacción y los costos de coordinación no superen los beneficios que genera. Por lo tanto, la conjunción de los factores anteriormente mencionados permitió una reducción sustancial de costos de transacción y de coordinación para las firmas en el mundo. Por otra parte, el planteamiento de (Porter, 1986) dio lugar a la comprensión de las actividades empresariales como una cadena de valor, en la cual la productividad de cada proceso es lo que finalmente genera valor para la compañía. En ese orden de ideas, las firmas buscan incrementar la productividad mediante el cambio tecnológico, la reducción de salarios o la capacitación de sus trabajadores (Jiménez \& Sierra, 2017). Así las cosas, las firmas han decidido externalizar (en otras empresas y otros países) las actividades que antes realizaban internamente, lo cual lleva a la fragmentación de la cadena de valor de un producto (Fally \& Hillberry, 2018; Gereffi \& Korzeniewicz, 1994). Estos procesos globales desarrollados por compañías en un entorno de mejora tecnológica constituyen el origen de las cadenas globales de valor (CGV). Debido a las implicaciones que esto tiene sobre diferentes ámbitos de la economía (salarios, empleo, producción, exportaciones, entre otros), la política comercial se ha enfocado en encontrar mecanismos óptimos que permitan facilitar la inserción en las CGV (Antràs \& De Gortari, 2017). Del mismo modo, las variaciones de la política comercial de otros países tienen implicaciones para todos los actores implicados en las CGV con dicho país (Blanchard, Bown \& Johnson, 2016), lo que ha puesto de manifiesto un interés creciente por los efectos del proteccionismo global.

La configuración de las CGV se encuentra influida por diversos factores, no solamente por consideraciones económicas. Cuando las firmas participan en CGV, se ven obligadas a permitir que otras compañías conozcan en profundidad sus necesidades, técnicas y procesos productivos, de modo que se puede poner en riesgo el know-how de la compañía. Por lo tanto, los derechos de propiedad en el propio país y en los socios son un factor que incide en la profundización de las CGV (Alfaro, Chor, Antràs \& Conconi, 2019). En ese sentido, la capacidad productiva de las firmas también es un factor relevante, puesto que se requiere al menos una base de conocimiento especializado para poder participar en las CGV (Hernández \& Pedersen, 2017).

En cuanto a Latinoamérica, se reconoce que la región no se encuentra suficientemente integrada en las CGV (Cadestin, Gourdon \& Kowlaski, 2016), aunque destacan los casos de México y su integración con América del Norte en virtud del TLCAN (Chiquiar \& Tobal, 2019), 
de Chile y su integración con países de la cuenca del Pacífico (OECD, 2015), y el de la industria electrónica y médica en Costa Rica (Monge-González \& Zolezzi, 2012). En general, se percibe el potencial que tienen las CGV para fomentar el desarrollo económico de la región y la oportunidad que existe de crear encadenamientos intrarregionales (Hernández, Martínez-Piva, \& Mulder, 2014). En contraposición, Asia ha logrado niveles elevados de inserción en $\mathrm{CGV}$, creando incluso una brecha frente a otros países en desarrollo, pues la exportación de bienes intermedios y de servicios permite participar en diversas industrias de varios países (Stare, Jaklič \& Knez, 2019; Yu \& Luo, 2018). No obstante, algunos países enfrentan estancamiento salarial debido a la dependencia de las actividades de exportación frente a empresas extranjeras, las cuales mantienen primordialmente producción de bienes con bajo valor agregado (Raj-Reichert, 2019). Los casos anteriores resultan ilustrativos, por cuanto permiten observar que las CGV presentan oportunidades, pero, a su vez, enfrentan desafíos una vez se ha logrado cierto nivel de integración.

Para el caso mexicano se han realizado numerosas investigaciones relacionadas con las CGV. Se reconoce que la inserción en los mercados globales se ha dado mediante la participación en CGV (Padilla-Pérez \& Villareal, 2017). Por lo tanto, Proméxico (2016) propone diferentes mecanismos de integración de las empresas mexicanas en las CGV y reco- noce la importancia que estas tienen en el desarrollo económico, especialmente en las regiones más industrializadas del país. Específicamente, el proceso de inserción se dio en tres etapas: primero, con la firma del TLCAN en 1994 se incrementó la participación en CGV, especialmente en manufacturas de alta cualificación; segundo, la fuerte competencia de China a partir de su ingreso a la OMC, y tercero, a finales de la década del 2000 aumentó su participación en manufacturas de baja cualificación (Chiquiar \& Tobal, 2019). Desde una perspectiva crítica, se observa que la inserción de México en las GCV ha generado dependencia de las empresas nacionales hacia las extranjeras (Crossa, 2017), de modo que se configuran condiciones que hacen vulnerable la economía a choques externos, tales como los acuerdos comerciales que generan desviación de comercio (Expósito, 2017) o las modificaciones de política comercial de los países socios, principalmente Estados Unidos (Salam, 2019). Los resultados para sectores específicos son variados. En el sector automotor se produjo la sofisticación de los servicios ofrecidos, así como roles más amplios en la cadena (Contreras, Carrillo, \& Alonso, 2012; Lampón, Cabanelas, \& Delgado, 2018). En el sector de la industria aeroespacial no se evidencian mejoras en términos de servicios ofrecidos ni desarrollo de capacidades de la fuerza de trabajo (Sandoval, Morales, \& Díaz, 2019), y en el sector TIC los resultados son mixtos, pues solo algunas empresas evidencian mejoras en su inserción y 
capacidades (Domínguez-Villalobos, Brown-Grossman, \& Carrera-Riva, 2017). No obstante, algunos autores plantean que estos problemas son causados por políticas públicas desacertadas y por el entorno institucional del país (Dougherty \& Reynaud, 2017; Sandoval, Morales, \& Díaz, 2019), lo cual es más notable cuando se realizan comparaciones frente a otros países con resultados superiores, como China (Gómez \& González, 2016). Metodológicamente, se encuentran propuestas útiles para lograr explicaciones de fenómenos específicos, pero el planteamiento metodológico no permite establecer comparaciones globales (Blyde, 2014; Dussel, 2018).

Las CGV se encuentran en casi todos los sectores de la economía, incluidos los servicios. No obstante, se concentran en el sector industrial, debido a la amplitud de los bienes intermedios que se utilizan en el proceso productivo. Esto es relevante, puesto que las exportaciones de manufactura son un elemento fundamental para el desarrollo económico (Marconi, Reis \& Araújo, 2016). Pero estos beneficios, por su naturaleza global, presentan una dependencia del comportamiento de la economía internacional, pues las CGV actúan como un transmisor de los fenómenos internacionales hacia la economía nacional (Lee \& Yi, 2018). Los cambios proteccionistas en la política comercial implican que el valor de las transacciones comerciales aumenta, lo que restringe el acceso a materias primas y encarece la exportación del bien luego de realizar el proceso y agregación de valor (Gangnes, Ma \& Assche, 2014). Así, resultan disminuidos los beneficios de las cadenas globales de valor, además de disminuir la confianza de los empresarios. Asimismo, la disminución de la producción -o el aumento de sus costos - tiene efectos negativos sobre el empleo, por cuanto las empresas se ven obligadas a disminuirlo o a incrementar la productividad por medio de capital, que sustituye al trabajo.

Este último punto requiere una elaboración adicional. Los avances en tecnologías que permiten la automatización de tareas físicas y de procesos cognitivos tienen impactos notables en el empleo, especialmente en el sector manufacturero (Brynjolfsson, \& McAfee, 2014). La robótica avanzada, el internet de las cosas y la inteligencia artificial permiten que las máquinas realicen tareas que previamente estaban reservadas a los trabajadores humanos. Como lo afirman (Tyson \& Lund, 2017), desde la crisis del 2008 las empresas en el mundo tuvieron que enfrentar un aumento de costos de producción y menores ventas con recortes de personal. Pero compensaron esto con el uso de tecnologías de automatización, que generaron un incremento en la productividad, lo que causó estancamiento en los salarios y la tasa de empleo. Además, ocasionan una concentración de los beneficios en las firmas que tienen acceso a tales tecnologías, lo que tiene incidencia en la disminución de la competencia y el incremento de la desigualdad Acemoglu \& Restrepo, 2016; Autor et al., 2017). 


\section{DATOS Y METODOLOGÍA}

La medición de las CGV ha presentado el inconveniente de no contar con bases de datos que compilaran sistemáticamente la información de múltiples países durante varios años. La mayoría de los análisis se realizan utilizando matrices insumo-producto con datos proporcionados por las oficinas nacionales de estadística de algunos países o bases de datos con información desglosada de la producción de empresas. Estas matrices pueden medir las CGV de dos maneras. Por una parte, presentar el contenido extranjero de la producción doméstica al tener en cuenta los insumos importados en la producción o en el total de los insumos importados (Amador \& Cabral, 2014). Por otra parte, la metodología de Hummels, Ishii y Yi (2001) estima el contenido importado de las exportaciones de un país. Esta última resulta más amplia en la medida en que incluye los insumos utilizados directa e indirectamente en el proceso productivo, pero más estrecha, puesto que contabiliza únicamente aquellos productos que se destinan a la exportación (Amador \& Cabral, 2014), aunque asegura que los resultados miden una integración en CGV más fuerte.

No obstante, estas presentan la dificultad de no aplicar las mismas metodologías en la obtención de datos y tampoco en el procesamiento de estos. Por lo tanto, en el año 2013, la Organización para la Cooperación y el Desarrollo Económico (OCDE) lanzó en conjunto con la Orga- nización Mundial del Comercio (OMC) la base de datos Trade in Value Added (TiVA, por sus siglas en inglés), que se basa en la información de las tablas Inter-Country Input-Output (ICIO, por sus siglas en inglés) las cuales recopilan datos de los sistemas de cuentas nacionales con la Clasificación Internacional Industrial Estándar (CIIU) versión 4. Al momento de su lanzamiento, comprendía 40 países con datos hasta el 2011, pero con la última actualización de los datos en diciembre 2018, se encuentran 64 países con datos disponibles hasta el 2015, y algunos hasta el 2016. Esta base de datos permite descomponer las exportaciones brutas totales o sectoriales de una economía según el contenido nacional y extranjero. También permite analizar el comercio de bienes intermedios. En general, posibilita medir la integración en CGV a través del análisis de las importaciones de bienes intermedios contenidas en las exportaciones de bienes (finales o no), y el valor doméstico añadido en las exportaciones de otros países. Esta base de datos se utiliza en el presente documento para analizar la integración de las manufacturas mexicanas en la economía global, así como en el análisis de los principales socios. El documento trabaja sobre los últimos datos disponibles según el indicador analizado.

Por último, es pertinente señalar que los valores que presenta la base de datos TiVA varían respecto a las fuentes tradicionales. Estas últimas se valoran según los precios de comprador, que 
incluyen los precios del productor, los márgenes comerciales y de transporte e impuestos no deducibles. Para poder utilizar los datos y cruzarlos en matrices de insumo producto, la OCDE los transforma en precios básicos, los cuales son la cantidad que recibe el productor por parte del comprador por una unidad de bien o servicio, excluyendo impuestos y cargos de transporte, pero contabilizando los subsidios.

En lo referente al análisis del empleo, se utiliza la base de datos Trade in eMployment (TiM, por sus siglas en inglés), la cual es publicada por la OCDE y es consistente con los datos publicados en TiVA. La base de datos presenta cómo el consumo en un país puede crear empleos en otros países a lo largo de la cadena de valor. En otras palabras, muestra el origen de la demanda que sostiene empleos en un país, revelando así la integración y la interdependencia en la economía global. La última versión de la base datos TiM corresponde al año 2018 y cuenta con información hasta el año 2015.

\section{Panorama comercial de México}

El comercio exterior de México se ha incrementado sostenidamente desde el año 2001, creciendo a una tasa anual promedio de $7 \%$ hasta alcanzar en el año 2018 exportaciones por valor de 450532 millones de dólares e importaciones por valor de 464268 millones de dólares.

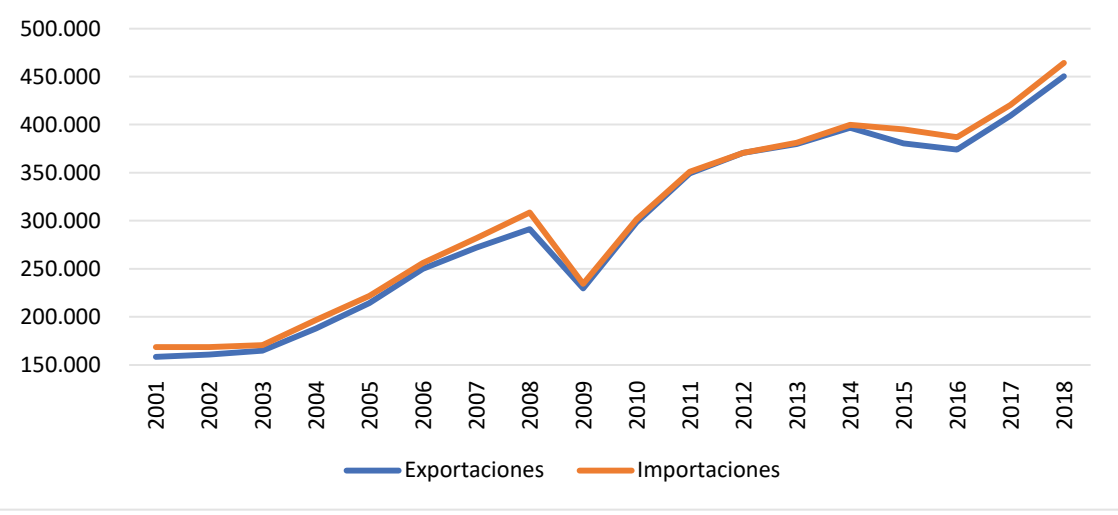

Figura 1. Comercio exterior de mercancías, México 2001-2018 (millones de dólares).

Fuente: elaboración propia con base en datos COMTRADE.

La balanza comercial indica el saldo neto en el comercio exterior de una economía. Es superavitario cuando las exportaciones son mayores que las importaciones, y deficitario en caso contrario. Ahora bien, los resultados de la balanza comercial deben interpretarse con cautela, puesto que únicamente 
hacen referencia al intercambio comercial de bienes y servicios en el ámbito internacional. Para el caso mexicano es pertinente analizar la balanza de pagos, dado que permite evidenciar el volumen del comercio internacional, así como la complejidad de dicha red entre México y Estados Unidos. Existen países con un déficit comercial durante varios años, que crecen económicamente a tasas elevadas, así como otros que cuentan con un gran superávit, pero tienen problemas económicos (Irwin, 2017). Analizar este indicador resulta pertinente por cuanto permite observar la evolución comercial en términos de crecimiento del volumen exportado e importado, tanto en términos generales como específicos respecto a otro socio comercial.

La Figura 2 presenta el resultado de la balanza comercial de bienes, en la que se evidencia que durante todo el periodo tiene un déficit comercial, que es bajo y estable y alcanza cerca de 13737 millones de dólares en el último año. No obstante, para el caso de los automóviles existe un superávit comercial por un valor superior a 60000 millones de dólares, lo que pone de manifiesto la relevancia de realizar un análisis en el campo industrial. Cabe resaltar que, entre las principales exportaciones del país, no se cuentan hidrocarburos ni materias primas, lo que demuestra cierto desarrollo de la industria mexicana. En ese sentido, las cifras de las importaciones de materiales básicos muestran altos niveles de importación de plástico, combustible, químicos, aluminio, hierro y caucho, pues son insumos necesarios para el desarrollo industrial que no se producen de forma suficiente para satisfacer la demanda local.

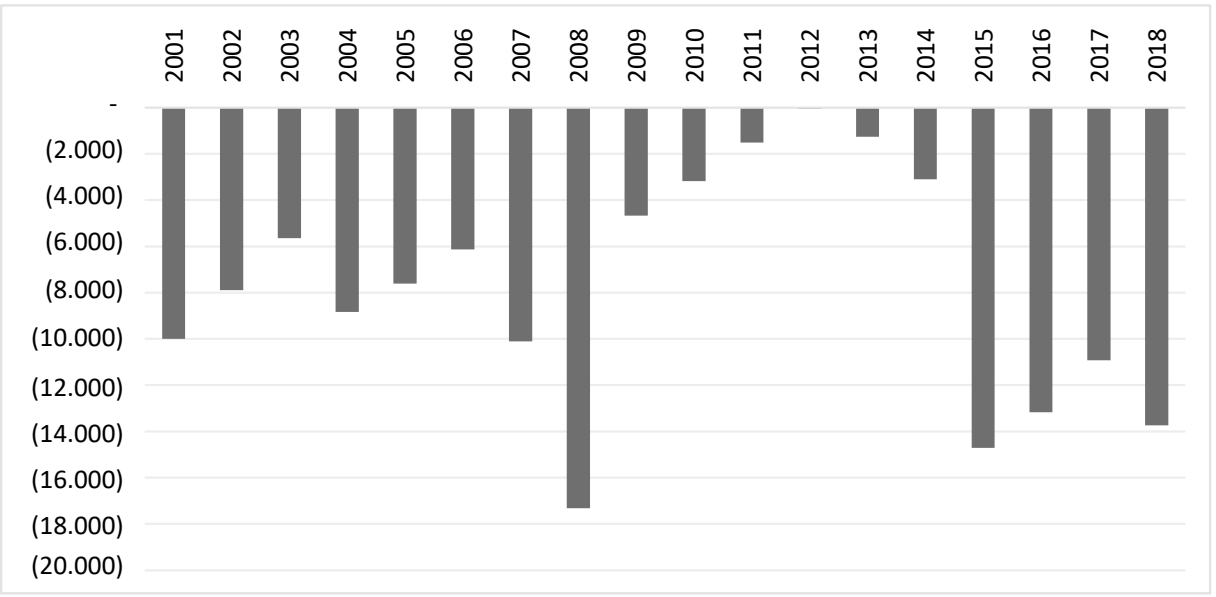

Figura 2. Balanza comercial de bienes, México 2001-2018 (millones de dólares).

Fuente: elaboración propia con base en datos COMTRADE. 
Brasil es la mayor economía de América Latina, pero México es el país que tiene mayor valor de exportaciones, $y$ según datos del 2018, duplica a Brasil. Un índice de participación tan elevado demuestra la dependencia económica que tiene México frente a sus exportaciones, pues representan casi el $40 \%$ del PIB. Adicionalmente, cuando se examina la concentración en socios comerciales, se encuentra que la mayoría (76\%) es destinada a Estados Unidos. Esta condición de dependencia hace que México sea especialmente vulnerable a cambios en la política comercial global y en la disrupción de los sistemas de producción. Luego de la crisis del 2008 muchas plantas de producción industrial, especialmente automotriz, cerraron en Estados Unidos, redujeron su producción o la trasladaron a países con menores costos como México. Esto explica el creciente superávit que registra esta rama de producción. Puesto que el sector manufacturero representa la mayor parte de las exportaciones de México (ver Figura 3), este documento se centra en su análisis.

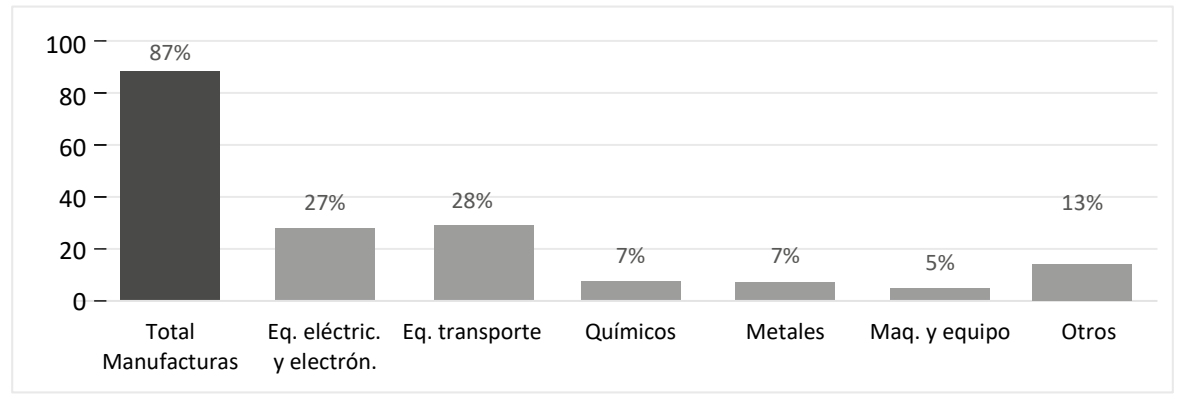

Figura 3. Participación de las exportaciones de manufactura en las exportaciones, México (promedio 2010-2015 y principales subsectores).

Fuente: elaboración propia con base en OCDE.

Por lo tanto, se evidencia que el sector manufacturero es el más importante dentro de las exportaciones mexicanas, y que más de la mitad de dichas exportaciones se encuentran concentradas en equipo eléctrico y en equipo de transporte. El siguiente apartado examina algunas características de la integración del sector de manufactura mexicano, enfatizando en la integración que presenta en el comercio internacional.
Para ello, se analiza el valor agregado nacional y extranjero, así como la participación de los bienes intermedios en la manufactura.

\section{Efectos en el comercio internacional}

La proliferación de las cadenas globales de valor (CGV) ha hecho que en la medición de exportaciones deba tenerse en cuenta el valor agregado al producto, 
más que el valor final del producto. Puesto que la medición actual de cifras de comercio internacional se basa en la declaración del valor (FOB/CIF) de la mercancía, los valores resultan distorsionados, dado que al país que realiza una parte del proceso productivo le otorga el valor total del producto. Por ejemplo, si Estados Unidos exporta autopartes y carrocerías a México para que las ensamble, cuando este último las exporte se contabiliza como si hubiera fabricado la totalidad del producto.

Esto tiene dos problemas principales, a saber: por un lado, sobreestima las cifras de comercio internacional; por otro lado, subestima la importancia del know-how al considerar que para fabricar un vehículo solamente se necesitan piezas y no conocimiento (Ghemawat \& Hout, 2017). Dado lo anterior, la exportación de manufacturas en México tiene que analizarse teniendo en cuenta una perspectiva que permita observar el valor agregado. Puesto que gran parte de estas hacen parte de cadenas globales de valor, las estadísticas brutas de exportación sobreestiman la participación.

En ese orden de ideas, es pertinente mencionar que la producción de mercancías manufacturadas -y el valor agregado- la realizan empresas nacionales y empresas extranjeras. Así las cosas, de cada USD 100 exportados por México, USD 54 corresponden a empresas nacionales y USD 46 a empresas extranjeras, tal como se observa en la Figura 4. Estos resultados permiten observar que las exportaciones mexicanas tienen un componente nacional y extranjero relativamente similar, lo que quiere decir que la industria mexicana tiene cierta dependencia de su producción manufacturera en empresas extranjeras.

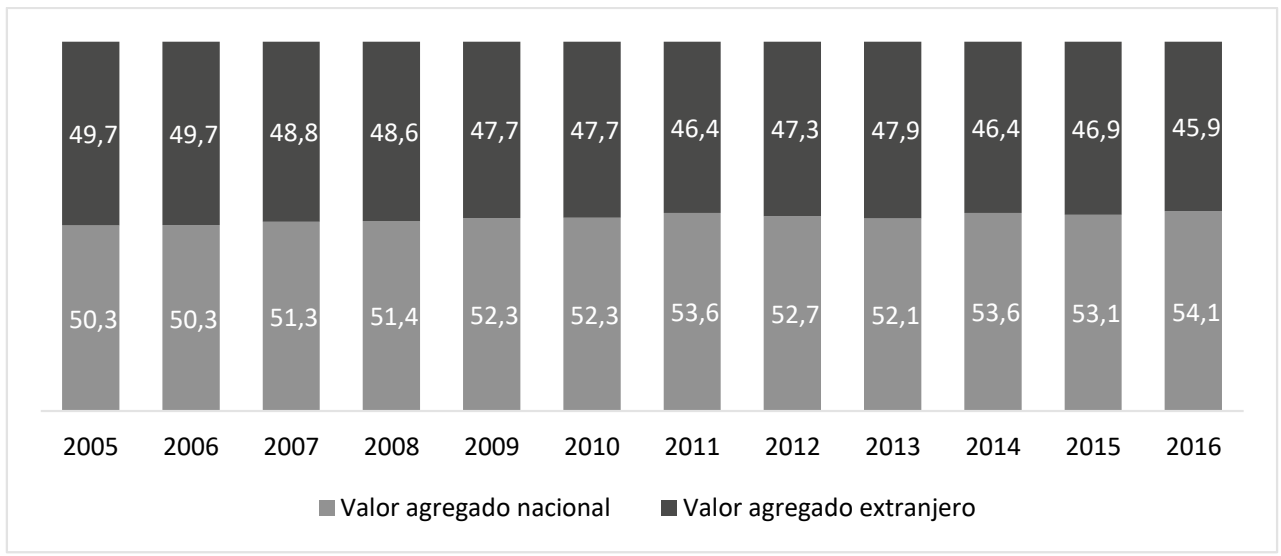

Figura 4. Valor agregado realizado por empresas nacionales y extranjeras, México porcentaje de exportaciones de manufactura (2005-2016).

Fuente: elaboración propia con base en datos TiVa compilados por OCDE y OMC (2016). 
Una medida de los niveles de integración de las economías en las cadenas globales de valor es la relevancia de los bienes intermedios en las importaciones, pues estos usualmente son utilizados para realizar un proceso de transformación que añada valor. Duran- te el periodo analizado, estas importaciones correspondieron al $58 \%$ del total de las importaciones manufactureras de México (Figura 5), lo cual indica que gran parte del comercio se lleva a cabo con el fin de desarrollar actividades de procesamiento.

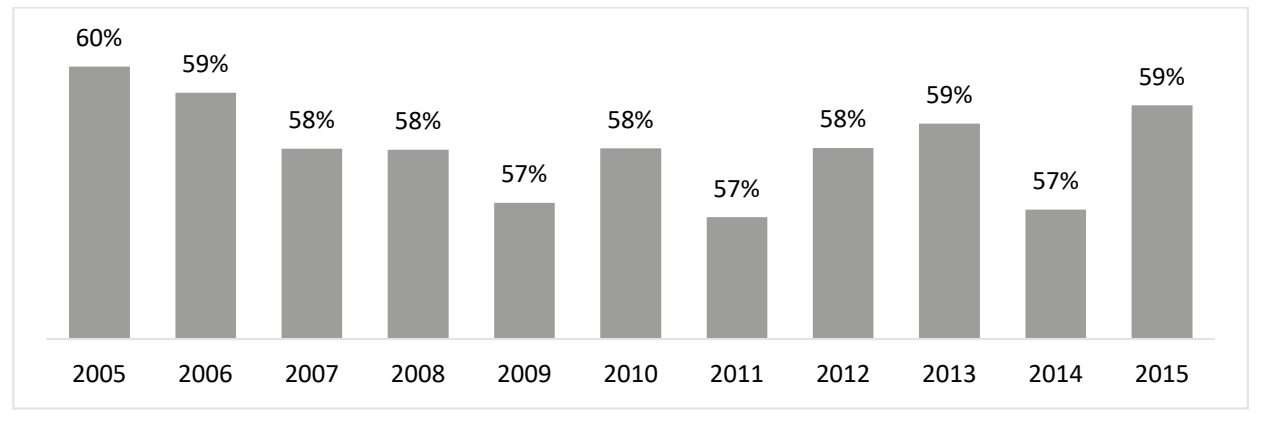

Figura 5. Importaciones de bienes intermedios. México, porcentaje de importaciones de manufactura (2005-2015).

Fuente: elaboración propia con base en datos TiVa compilados por OCDE y OMC (2016).

Como mencionan Bas y Strauss-Khan (2014), la importación de bienes intermedios aumenta la productividad e incentiva las exportaciones, de modo que se observa una estrecha relación interdependiente entre las importaciones de productos intermedios y las exportaciones de productos finales. Ahora bien, para comprender la profundidad de la participación de México en las cadenas globales de valor, cabe cuestionar qué porcentaje de dichas importaciones de bienes intermedios vuelven a exportarse posteriormente, ya sea en productos intermedios o finales. Mientras que el promedio de los países del TLCAN es de $26 \%$, el de México es de $58 \%$, superando incluso ligeramente a los países industriales de ASEAN (55\%). Desde el inicio de este siglo, la participación de México en las CGV del TLCAN ha permanecido constante, posiblemente por la maduración de las cadenas que iniciaron luego de la firma del tratado en 1994. Esto es un indicativo de la profundidad de las cadenas de valor, dado que más de la mitad de los insumos importados se utilizan en productos -terminados o no- que vuelven a ser exportados posteriormente. Por último, vale resaltar la participación de bienes intermedios chinos, pues para el 2015 fue el $21 \%$ del total, así como de Corea del Sur y Japón ( $4 \%$ cada uno), lo que hace que el Sudeste Asiático provea casi el 30\% de los bienes intermedios requeridos por México. 
Otro punto de vista que confirma la profundidad de las CGV en México es la participación de bienes intermedios y bienes finales que exporta. Del total de esas exportaciones, se observa que México es el país que más destina el valor agregado de sus exportaciones hacia Estados Unidos: 76\% en promedio durante el 2001-2011, seguido por Canadá $(67 \%)$ y Colombia en el tercer lugar (37\%). En promedio, el 15\% del valor de las mercancías que México exporta fue hacia Estados Unidos. Esto pone de manifiesto la elevada dependencia de México del mercado estadounidense.

\section{Efectos en la economía nacional}

Los resultados previos permiten determinar la distribución del valor agregado entre empresas nacionales, es decir, lo que el país es capaz de producir por sí mismo, y el valor agregado dado por empresas extranjeras, esto es, los requerimientos del país en términos de capacidades productivas del extranjero. Así pues, se evidencia que el aparato producto de México requiere del libre flujo comercial para desarrollar sus actividades económicas, por cuanto una proporción notable de su producción hace parte de cadenas de valor, lo que supone la importación de bienes intermedios, la transformación en México por parte de empresas locales y extranjeras, y la posterior exportación de las mercancías. Por lo tanto, las decisiones de política comercial tienen impactos en la economía doméstica y el empleo de México.

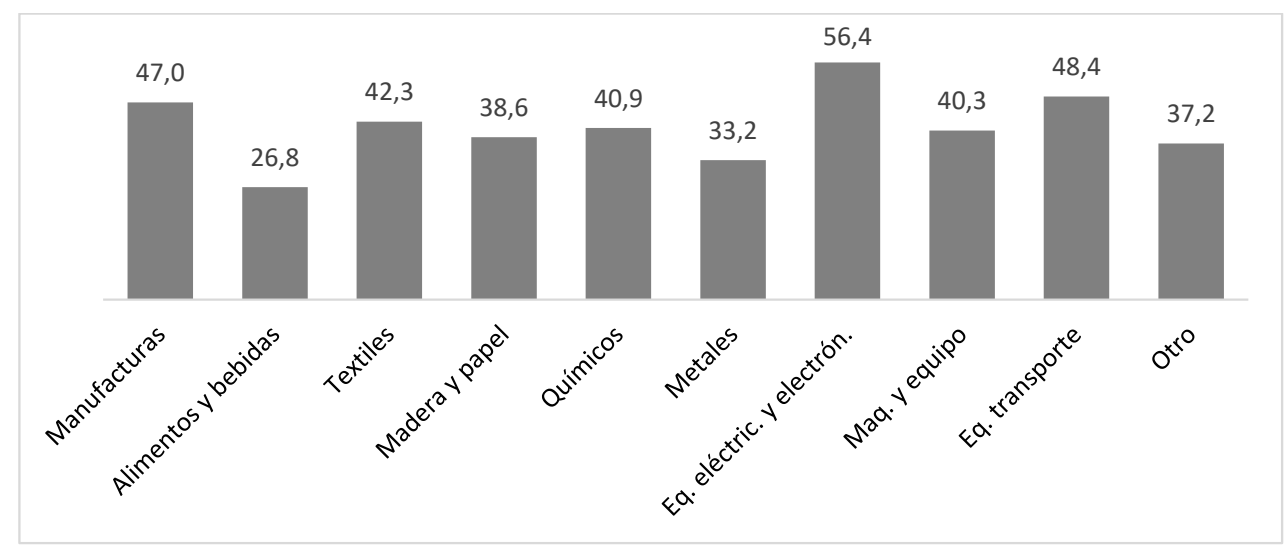

Figura 6. Valor agregado realizado por empresas extranjeras, México porcentaje de exportaciones (promedio 2011-2015).

Fuente: elaboración propia con base en datos TiVa compilados por OCDE y OMC (2016).

Esto indica los requerimientos de capacidades extranjeras para la exportación de manufacturas en México: de cada
USD 100 exportados, se requiere una participación de USD 47 en contenido extranjero. Los requerimientos son 
distintos según el sector manufacturero que se examine. Para el caso de la fabricación de equipo eléctrico y equipo de transporte, la participación es considerable por parte de empresas foráneas, principalmente provenientes de Canadá y Estados Unidos. Como se observa en la Figura 6, la importancia de las exportaciones manufactureras en México es elevada, pues gran parte de sus exportaciones se concentran en dicho sector, siendo las ramas más representativas el equipo eléctrico y el de transporte.

Ahora bien, para determinar el impacto de estos sectores, es posible medir el dinamismo exportador y la relevancia que tiene en la economía nacional de México, como se observa en la Figura 7. El dinamismo exportador se calcula teniendo en cuenta el promedio del crecimiento anual de las exportaciones de cada sector durante el periodo 20052015. Por su parte, el impacto sobre la economía nacional se mide con el valor agregado nacional como porcentaje de las exportaciones del año 2015. Finalmente, el tamaño de las burbujas corresponde al valor exportado durante el año 2015. Los cuadrantes se establecen según el promedio del valor de cada variable.

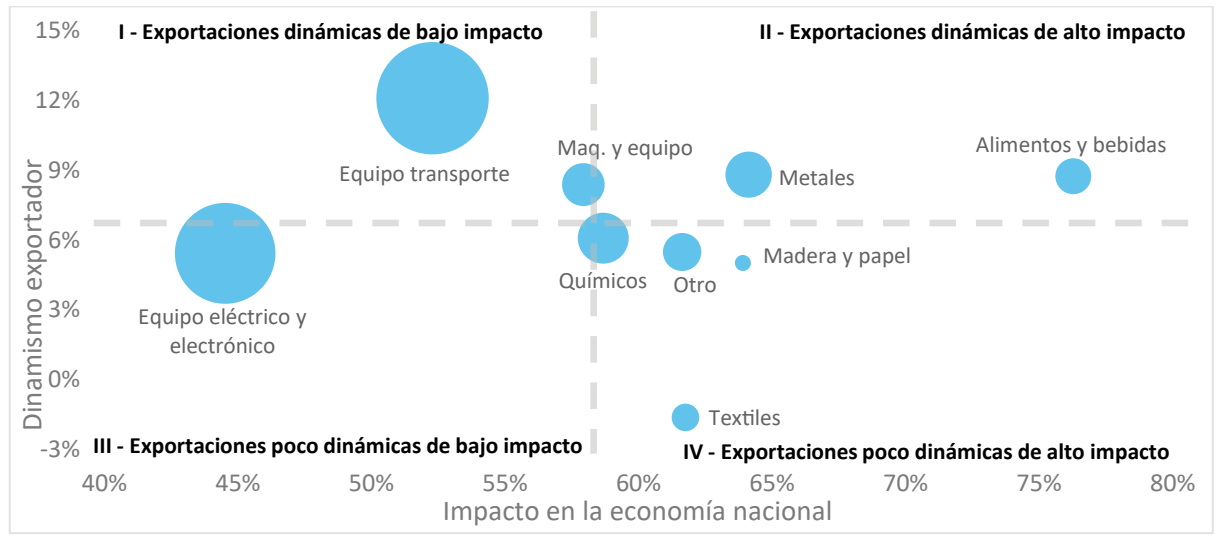

Figura 7. Dinamismo de las exportaciones manufactureras e impacto sobre la economía nacional de México.

Fuente: elaboración propia con base en OCDE.

En primera instancia se encuentra que los dos sectores con mayor valor de exportaciones son equipos de transporte y equipos eléctricos (entre 107590 millones de dólares y 86405 millones de dólares); los sectores con un valor intermedio de exportaciones son metales y químicos (entre 18139 millones de dólares y 22144 millones de dólares) y, los sectores con menor valor de exportaciones son textiles y papel y madera (entre 6437 millones de dólares y 2181 millones de dólares). Ahora bien, considerando que el promedio del crecimien- 
to de las exportaciones de manufacturas fue de $6.5 \%$ durante el periodo 2000 2011, y que el valor agregado nacional como porcentaje de las exportaciones de manufactura es de 59\% en el año 2011, es posible segmentar en cuatro secciones la figura, según si el dinamismo y el impacto están por encima o por debajo del promedio mencionado previamente.

En lo que respecta al cuadrante 1 (exportaciones dinámicas de bajo impacto), únicamente se ubica el sector de equipo de transporte, el cual ha tenido un crecimiento promedio notable (12.1\%), y poco más del $50 \%$ en valor agregado doméstico. Los resultados concuerdan con las principales críticas al modelo de integración mexicano que se plantean en la literatura (Crossa, 2017; Gómez \& González, 2016), pues la industria se destaca por su crecimiento, pero no es así con los niveles salariales ni el número de empleos, elemento que se analiza en profundidad en la siguiente sección y resulta evidente en la Figura 10.

En el caso del cuadrante 2 (exportaciones dinámicas de alto impacto), se encuentran dos sectores que representan aquellos que tienen un mayor potencial de crecimiento y un impacto más grande en la economía de México. Cabe mencionar que ninguno corresponde a los grandes sectores tradicionales y que sus valores exportados son medianos. En particular, el sector de alimentos y bebidas destaca por su mayor impacto sobre la economía nacional (76\%); empero, esta cifra debe ser tomada con cautela, pues el sector agrícola por su propia naturaleza implica necesariamente trabajo doméstico. En lo referente al sector de metales, este es el segundo con más dinamismo $(8.8 \%)$ después del equipo de transporte, aunque ha presentado una caída desde el 2013 hasta el 2018.

Para el cuadrante 3 (exportaciones poco dinámicas de bajo impacto), se encuentra el sector de equipo eléctrico y electrónico con crecimiento moderado (5.4\%) y un impacto relativamente bajo (45\%), aunque de ninguna manera desdeñable, dada la cantidad de personas empleadas en el sector (casi 240 000). No obstante, como se evidencia en la Figura 10, la productividad del sector ha aumentado constantemente al tiempo que el empleo ha disminuido, sugiriendo así mejoras en el capital humano y uso de tecnologías. En lo que refiere al cuadrante 4 (exportaciones dinámicas de bajo impacto), allí se encuentra el mayor número de sectores: madera y papel, textiles, y químicos. Este último presenta un dinamismo notable (6.1\%), así como un impacto en la economía del $59 \%$, lo que lo convierte en el sector de más crecimiento de aquellos con valores moderados de exportación.

Como resultado general, es posible observar que los sectores con mayor valor de exportaciones $-\mathrm{y}$ aquellos que más empleos generan- tienen un valor agregado inferior a la media. Por otro lado, destacan varios sectores que tienen un valor de exportación menor, pero debido a su elevada tasa de creci- 
miento presentan un potencial notable, así como un impacto considerable en la economía al generar un valor agregado superior a la media del sector de manufacturas. Ahora bien, otra manera de estudiar la relevancia de los sectores de manufactura en la economía mexicana es observar el empleo.

\section{Empleo en los sectores de manufactu- ra en México}

Los encadenamientos productivos generados por las CGV hacen que los puestos de trabajo de la economía nacional se tornen interdependientes de los mercados externos, pues algunos puestos de trabajo existen únicamente para satisfacer la demanda de consumidores extranjeros. En ese orden de ideas, es pertinente analizar cuál es el nivel de dependencia de estos puestos de trabajo en general, y en el sector manufacturero en particular. De igual modo, el nivel de integración con cada país es diferente, de manera que también cabe examinar el origen de la demanda de las mercancías con valor agregado. Dadas las recientes tendencias tecnológicas de automatización para incrementar la productividad de las firmas, se formulan algunos planteamientos acerca del impacto que esto ha tenido en el empleo.

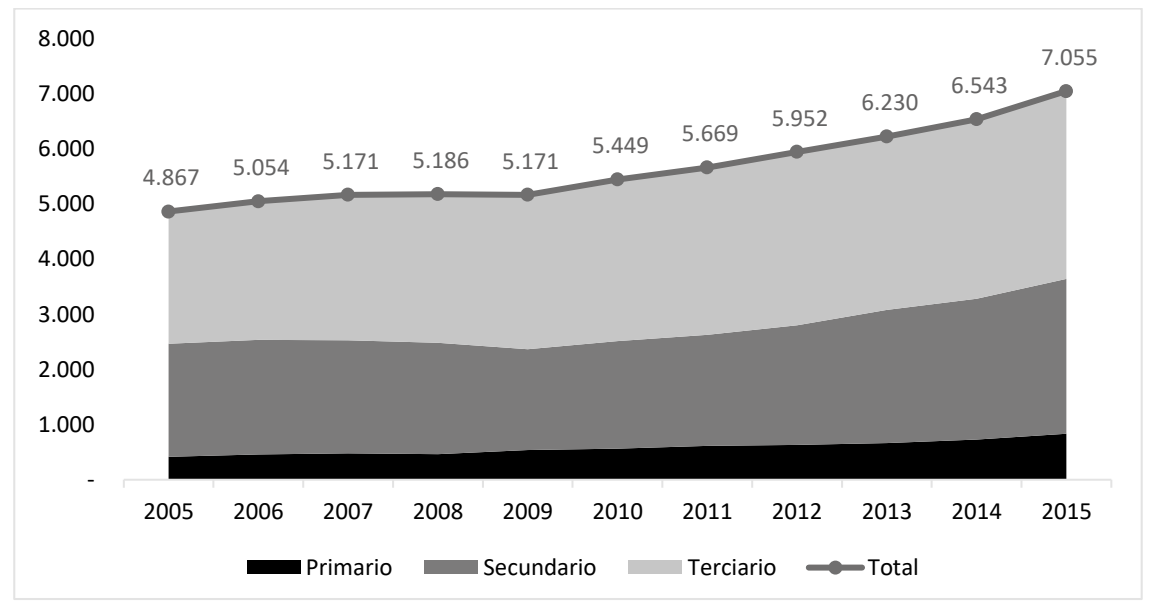

Figura 8. Empleos en sectores ligados a la exportación, México (2005-2015).

Fuente: elaboración propia con base en OCDE, TiM Indicators.

En México, más de siete millones de empleos son dependientes de actividades de exportación, lo que quiere decir que el 19\% de los empleos mexicanos dependen de la demanda final externa ${ }^{1}$. Se evidencia que el sector

1 Según datos de la OCDE (trade in employment statistics), en el año 2015 había 37.5 millones de empleos en México. 
servicios es el que más emplea personas en actividades orientadas a la exportación, pues representa casi la mitad (48\%) de dichos trabajos. Para el caso del sector manufacturero, se encuentra que para el 2015 generaba 2811 puestos de trabajo, lo que equivale al $40 \%$ del total. El sector primario, por su parte, emplea el $12 \%$ de las personas ubicadas en actividades de exportación. No obstante, la medición debe ser interpretada con cierta cautela, pues la base de datos utilizada incorpora exportaciones solo a las 61 principales economías, de modo que algunos datos resultarán omitidos. Aunque la cifra se encuentra por debajo de otros países en desarrollo, como lo evidencia Mendoza (2010), es importante resaltar que estos empleos son formales y tienen salarios más elevados que aquellos en sectores no orientados a la exportación, siendo esto consecuente con lo presentado en el marco teórico de este trabajo.

Para observar los países que más generan la demanda del valor agregado de estos productos en México, se tienen los datos de la Figura 9. Los países miembros del TLCAN, como era de esperar, generan la mayoría de los puestos de trabajo dedicados a la exportación de manufacturas, pues la cercanía geográfica y la integración industrial que permitió el acuerdo hacen de estos países los principales socios. Esto es congruente con las estadísticas de importación de bienes intermedios, puesto que el $49 \%$ de los bienes intermedios proceden de estos dos países.
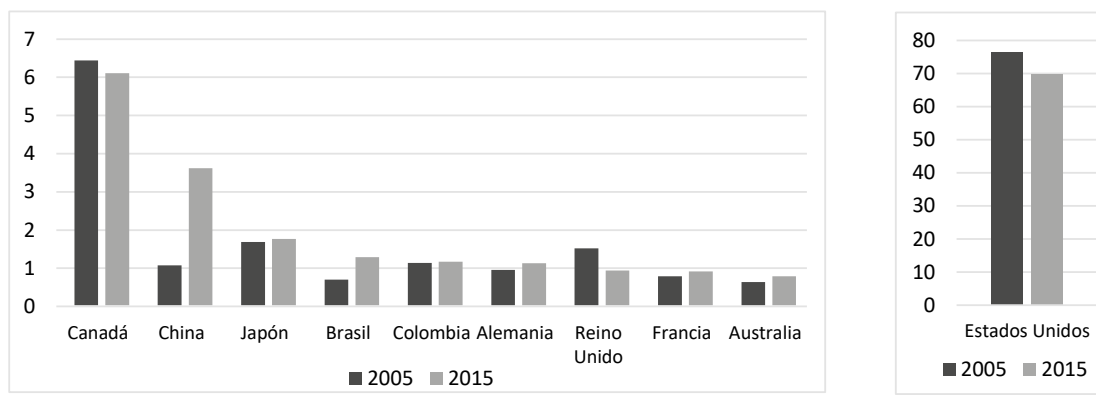

Figura 9. Principales 10 socios comerciales que generan la demanda de valor agregado en manufacturas: comparación 2005 y 2015 (porcentaje de la demanda externa).

Fuente: elaboración propia con base en datos OCDE.

A partir de lo anterior, se considera lo siguiente: primero, la concentración en Estados Unidos es bastante alta -supera la media de los países OCDE-, aunque ha mostrado una reducción mínima, que representa el $70 \%$ de la demanda para el 2015; esto se debe principalmente al incremento de la participación de otros países, pues al observar las cifras en términos absolutos, han aumentado. 
Segundo, es notable el aumento de la demanda externa por parte de China, que apenas alcanzaba $0.9 \%$ en 2005 . Cabe destacar la relevancia de la cuenca del Pacífico para México, pues entre sus principales socios se encuentran China, Japón y Australia. Tercero, los países de América Latina presentan un incremento en la participación: Brasil y Colombia ocupan el cuarto y quinto lugar, respectivamente. Además, el incremento también se presenta en otros países de la región como Chile, Argentina, Perú y Ecuador, lo que muestra de alguna manera los cambios en la integración de la producción de México y de América Latina.

Ahora bien, cuando se analizan los empleos manufactureros, surge una tendencia llamativa. Como se observa en la Figura 9, el valor exportado del total de manufacturas y de su principal sector (equipos de transporte) ha aumentado sostenidamente durante el periodo 2000-2011. No obstante, el número de empleos en ambos sectores ha disminuido. Al inicio del periodo, la proporción empleo/exportación en el total de manufacturas era de 38 (esto es, se requerían 38 trabajadores para exportar un millón de dólares), mientras que en el 2015 la cifra se redujo a $20^{2}$. Hay que aclarar que el empleo total en el sector ha aumentado en términos absolutos (5\% del 2005 al 2015), pero lo importante es observar el comportamiento en términos relativos frente a los niveles de producción. Por su parte, en el sector de manufacturas, dicha proporción se redujo de 12 a 7 durante el periodo examinado. Así las cosas, la producción (exportación) ha aumentado más rápido que las tasas de empleo, lo que sugiere que estos sectores (intensivos en capital) están aumentando el uso de tecnología en su proceso productivo (Brynjolfsson, \& McAfee, 2014).

El quiebre observado en la crisis económica de 2008 resulta normal en estadísticas globales, dada la afectación de los mercados; sin embargo, a partir de entonces se inició una tendencia a reemplazar los empleos perdidos con máquinas o complementar los existentes con estas, haciendo que la recuperación posterior del empleo fuera difícil, al tiempo que se lograban notables aumentos en la producción (Tyson \& Lund, 2017).

2 Cabe recordar que estos datos son los empleos ligados a actividades de exportación. De cualquier modo, la tendencia se mantiene cuando se observa el valor de producción total (incluyendo la destinada al mercado local) y el número total de empleos. Ver informe de Proméxico y estadísticas de INEGI: https://www.promexico.mx/documentos/biblioteca/the-mexican-automotive-industry.pdf 


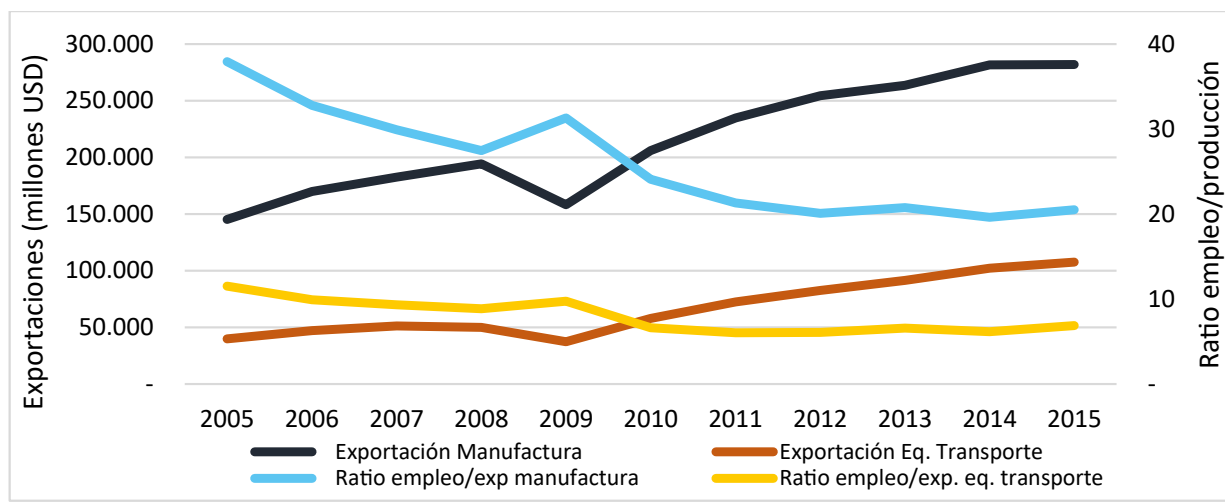

Figura 10. Exportaciones de equipos eléctricos y de transporte, y proporción de empleo (México 2005-2015).

Fuente: elaboración propia con base en datos OCDE.

Este comportamiento pone de manifiesto el segundo desafío que enfrenta la economía mexicana: la reducción de empleos en la manufactura como consecuencia del cambio tecnológico y la automatización (Guthrie, 2017). En el sector automotor existen varios trabajos que pueden ser reemplazados por máquinas o bien pueden incrementar su productividad haciendo uso de estas. En cualquier caso, o destruyen empleos o impiden que los empleos se incrementen, pues la productividad pasa de depender del trabajo a ser también un resultado del cambio tecnológico (Chui, Manyika, \& Miremadi, 2016). En ese sentido, compañías que proveen servicios de automatización han visto sus ventas duplicarse entre el 2016 y el 2017. De acuerdo con el Boston Consulting Group (BCG, 2015) México es la economía emergente que más ha avanzado en procesos de automatización, previendo que hacia el año 2025 aproximadamente el $30 \%$ de las tareas serán desarrolladas por máquinas, frente a un $10 \%$ como promedio global.

Ahora bien, puesto que los salarios de la industria automotriz en México son inferiores a los de los países desarrollados (Center for Automotive Research, 2016), las máquinas aún no tienen el costo suficientemente bajo como para reemplazar a este tipo de trabajadores (Guthrie, 2017). Por lo tanto, las empresas están utilizando las máquinas no tanto para reemplazar trabajos ya existentes, como para aumentar la productividad de dichos trabajadores, puesto que les permiten automatizar algunas tareas rutinarias, así como aumentar sus capacidades productivas - por ejemplo, moviendo más peso con una máquina más potente- . Esto se hace evidente al observar que los empleos no han disminuido en términos absolutos, lo que sugiere que no se están destruyendo puestos de trabajo, sino aumentando la productividad de los existentes. En 
cualquier caso, es una tendencia que merece mayor atención.

\section{CONCLUSIONES}

Las manufacturas tienen una relevancia notable en la economía mexicana, puesto que representan la mayor parte de las exportaciones de bienes, así como el segundo sector que más emplea personas en trabajos de exportación. De igual modo, depende de las manufacturas el proceso de inserción mexicano en las cadenas globales de valor. Se encuentra que el sector manufacturero debe ser analizado desagregándose en el campo industrial, lo que permite revelar que algunos sectores (como el automotor) que tienen un rápido crecimiento en las exportaciones, no necesariamente generan un alto grado de valor agregado. Asimismo, los resultados muestran que, para dicho sector, los aumentos en productividad no están asociados a mayores empleos ni a mejores salarios, lo que, apoyándose en literatura reciente, sugiere que hay incidencia del uso de tecnologías en el proceso productivo.

El análisis de las CGV mexicanas permite inferir dos desafíos. Dada la dependencia de las exportaciones y los empleos ligados a estas en México, los cambios en las políticas comerciales de Estados Unidos constituyen el primer desafío que enfrenta la economía mexicana, puesto que afecta las cadenas globales de valor de las que dependen miles de empleos en el país. Por otro lado, las cifras de empleo evidencian que el incremento en la productividad de la industria automotriz mexicana se debe más al aumento en la tecnología que en el capital humano, de modo que los empleos $-\mathrm{y}$ sus salarios- crecen en una proporción menor a la productividad y al valor exportado.

A partir de este estudio surgen cuestiones para explorar en futuras líneas de investigación. Primero, sería interesante analizar estas características en el sector servicios, pues los nuevos datos proporcionados por la OCDE permiten observar la importancia de los sectores frente al comercio exterior, un asunto que no era posible medir con los datos tradicionales de empleo, pues no tienen en cuenta el valor agregado de la producción enfocada en la exportación. Segundo, analizar la dinámica laboral, pues desde 1995 la proporción de empleos en sectores de exportación no ha variado: en el año 2011 continuaba siendo $16 \%$, una situación que no cabría esperar, dados los efectos de la integración económica mexicana. Tercero, sería conveniente examinar las oportunidades que presentan las cadenas globales de valor para los recursos naturales en América Latina, en consonancia con los postulados seminales de Tello (2017). Finalmente, resultaría pertinente investigar acerca de los efectos de la automatización en el mercado laboral mexicano, enfatizando en el sector automotor; trabajos similares se han hecho para Estados Unidos por Acemoglu y Restrepo (2017). 


\section{AGRADECIMIENTOS}

Los autores agradecen los comentarios de los dos evaluadores anónimos, así como las recomendaciones dadas por el editor.

\section{REFERENCIAS}

Acemoglu, D. \& Restrepo, P. (2016). The Race Between Machine and Man: Implications of Technology for Growth, Factor Shares and Employment. NBER Working Paper, (22252). Doi: https://doi.org/10.3386/w22252

Acemoglu, D. \& Restrepo, P. (2017). Robots and Jobs: Evidence from US Labor Markets. NBER Working Paper, (23285). Doi: https://doi.org/10.2139/ssrn.2940245

Aichele, R. \& Heiland, I. (2018). Where is the Value Added? Trade Liberalization and Production Networks. Journal of International Economics, 115, 130-144. Doi: 10.1016/j.jinteco.2018.09.002

Alfaro, L., Chor, D., Antràs, P. \& Conconi, P. (2019). Internalizing Global Value Chains: A Firm-Level Analysis. Journal of Political Economy, 127(2), 508-599. Doi: $10.1086 / 700935$

Amador, J. \& Cabral, S. (2014). Global Value Chains: A Survey of Drivers and Measures. Journal of Economic Surveys, 30(2), 278-301. Doi: doi.org/10.1111/ joes. 12097

Antràs, P. \& de Gortari, A. (2017). On the Geography of Global Value Chains. NBER Working Paper, (23456). Doi: https://doi.org/10.3386/w23456

Autor, D., Dorn, D., Katz, L. F., Patterson, C., \& Van Reenen, J. (2017). The Fall of the Labor Share and the Rise of Superstar Firms. NBER Working Paper, (23396). Doi: https://doi.org/10.3386/w23396

Baldwin, R. (2016). The Globotics Upheaval: Globalisation, Robotics and the Future of Work. New York: Oxford University Press.

Baldwin, R. (2019). The Great Convergence: Information Technology and the New Globalization. Cambridge: The Belknap Press of Harvard University Press. 
Bas, M. \& Strauss-Khan, V. (2014). Does Importing more Inputs Raise Exports? Firm-level Evidence from France. Review of World Economics, 150(2), 241-275. Doi: 10.1007/s10290-013-0175-0

Blanchard, E. J., Bown, C. P. \& Johnson, R. C. (2016). Global Supply Chains and Trade Policy. National Bureau of Economic Research, NBER Working Paper, (21883). Doi: https://doi.org/10.1596/1813-9450-7536

Blyde, J. S. (2014). The Participation of Mexico in Global Supply Chains: The Challenge of Adding Mexican Value. Applied Economics Letters, 21(7), 501-504. Doi: $10.1080 / 13504851.2013 .870647$

Boston Consulting Group -BCG-. (2015). The Robotics Revolution: The Next Great Leap in Manufacturing. BCG.

Brynjolfsson, E. \& McAfee, A. (2014). The Second Machine Age. New York \& London: W. W. Norton \& Co.

Cadestin, C., Gourdon, J. \& Kowlaski, P. (2016). Participation in Global Value Chains in Latin America. OECD Trade Policy Papers, (192). Doi: 10.1787/18166873

Center for Automotive Research -CAR-. (2016). The Growing Role of Mexico in the North American Automotive Industry. CAR.

Chiquiar, D. \& Tobal, M. (2019). Global Value Chains in Mexico: A Historical Perspective. Banco de México Working Papers, (2019-06).

Chui, M., Manyika, J. \& Miremadi, M. (2016). Where Machines could Replace Humans and where they can't (yet). McKinsey Quarterly. Retrieved from https://www.mckinsey.com/business-functions/digital-mckinsey/our-insights/ where-machines-could-replace-humans-and-where-they-cant-yet

Coase, R. H. (1937). The Nature of the Firm. Economica, 4(16), 386-405. Doi: 10.1111/j.1468-0335.1937.tb00002.x

Contreras, O. F., Carrillo, J. \& Alonso, J. (2012). Local Entrepreneurship within Global Value Chains: A Case Study in the Mexican Automotive Industry. World Development, 40(5), 1013-1023. Doi: 10.1016/j.worlddev.2011.11.012 
Crossa, M. (2017). Cadenas globales de valor en la industria del automóvil: la ilusión desarrollista o el desarrollo del subdesarrollo en México. Cuadernos de Economía Crítica, 3(6), 71-100

Domínguez-Villalobos, L., Brown-Grossman, F. \& Carrera-Riva, S. (2017). Mexico: Insertion of ICT Services in Global Value Chains, Capabilities and Public Policy. International Journal of Value Chain Management, 8(2), 117-134. Doi: 10.1504/IJVCM.2017.085482

Dougherty, S. \& Reynaud, J. (2017). Boosting Productivity in Mexico through Integration into Global Value Chains. OECD Economics Department Working Papers, (1376). Doi: 10.1787/18151973

Dussel, E. (2018). Cadenas globales de valor: metodología, teoría y debates. México: UNAM

Expósito, A. (2017). El shock externo del Acuerdo Transatlántico para el Comercio y la Inversión para la manufactura mexicana. ¿Cómo afectaría a sus exportaciones hacia los Estados Unidos? Trimestre Económico, 84(333), 237-254. Doi: https://doi.org/10.20430/ete.v84i333.268

Fally, T. \& Hillberry, R. (2018). A Coasian Model of International Production Chains. Journal of International Economics, 114, 299-315. Doi: 10.1016/j. jinteco.2018.07.001

Feenstra, R. (1998). Integration of Trade and Disintegration of Production in the Global Economy. The Journal of Economic Perspectives, 12(4), 31-50. Doi: 10.1257/jep.12.4.31

Gangnes, B. S., Ma, A. C. \& Assche, A. V. (2014). Global Value Chains and Trade Elasticities. Economic Letters, 124(3), 482-486. Doi: 10.1016/j.econlet.2014.07.018

Gereffi, G. \& Korzeniewicz, M. (1994). Commodity Chains and Global Capitalism. United States: Westport.

Ghemawat, P. \& Hout, T. (2017). ¿Pueden las empresas chinas conquistar el mundo? Foreign Affairs Latinoamérica, 17(2), 108-118.

Gómez, C. \& González, J. (2016). La presencia de China y México en las cadenas globales de valor. Una perspectiva crítica. Revista CIMEXUS, 11(1), 67-85. 
Guthrie, A. (2017). Trump Talk Aside, Auto Manufacturing and Automation Are Booming in Mexico. MIT Technology Review. Retrieved from https:// www.technologyreview.com/s/608220/trump-talk-aside-auto-manufacturing-and-automation-are-booming-in-mexico/

Hernández, R. A., Martínez-Piva, J. M. \& Mulder, N. (2014). Global Value Chains and World Trade. Santiago de Chile: ECLAC.

Hernández, V. \& Pedersen, T. (2017). Global Value Chain Configuration: A Review and Research Agenda. BRQ Business Research Quarterly, 20(2), 137-150. Doi: 10.1016/j.brq.2016.11.001

Hummels, D., Ishii, J. \& Yi, K. M. (2001). The Nature and Growth of Vertical Specialization in World Trade. Journal of International Economics, 54(1), 75-96. Doi: 10.1016/S0022-1996(00)00093-3

Irwin, D. A. (2017). La verdad acerca del comercio. Foreign Affairs Latinoamérica, $17(2), 119-128$.

Jiménez, Y. \& Sierra, Y. (2017). La inserción internacional en cadenas globales de valor. Economía y Desarrollo, 158(2), 189-205.

Krugman, P. (1979). Increasing Returns, Monopolistic Competition and International Trade. Journal of International Economics, 9(4), 469-479. Doi: 10.1016/0022-1996(79)90017-5

Krugman, P. (1991). Increasing Returns and Economic Geography. Journal of Political Economy, 99(3), 483-499. Doi: 10.1086/261763

Lampón, J. F., Cabanelas, P. \& Delgado, J. A. (2018). Claves en la evolución de México dentro de la cadena de valor global de la industria de autopartes. El caso del Bajío. El Trimestre Económico, 85(339), 483-514. Doi: https://doi.org/10.20430/ ete.v85i339.259

Lee, E. \& Yi, K-M. (2018). Global Value Chains and Inequality with Endogenous Labor Supply. Journal of International Economics, 115, 223-241. Doi: 10.1016/j. jinteco.2018.09.006

Marconi, N., Reis, C. F. \& Araújo, E. C. (2016). Manufacturing and Economic Development: The Actuality of Kaldor's First and Second Laws. Structural Change and Economic Dynamics, 37, 75-89. Doi: 10.1016/j.strueco.2015.12.002 
Monge-González, R. \& Zolezzi, S. (2012). Insertion of Costa Rica in Global Value Chains: A Case Study. IDB Working Paper Series, (IDB-WP-373).

OECD. (2015). Diagnostic of Chile's Engagement in Global Value Chains. Paris: OECD Publishing.

Padilla-Pérez, R. \& Villareal, F. G. (2017). Structural Change and Productivity Growth in Mexico, 1990-2014. Structural Change and Economic Dynamics, 41, 53-63. Doi: 10.1016/j.strueco.2017.02.002

Porter, M. E. (1986). Competition in Global Industries. Boston: Harvard Business School Press.

Proméxico. (2016). Cadenas globales de valor: un modelo de integración para las compañias mexicanas. Ciudad de México: Proméxico.

Raj-Reichert, G. (2019). Global Value Chains, Contract Manufacturers, and the Middle-Income Trap: The Electronics Industry in Malaysia. The Journal of Development Studies, 56(4), 698-716. Doi:10.1080/00220388.2019.1595599

Salam, K. (2019, 7 jun.). FP's Guide to the U.S.-Mexico Trade War. Foreign Policy. Retrieved from https://foreignpolicy.com/2019/06/07/ fps-guide-to-the-u-s-mexico-trade-war/

Sandoval, S., Morales, M. A. \& Díaz, H. E. (2019). Estrategia de escalamiento en las cadenas globales de valor: el caso del sector aeroespacial en México. EntreCiencias, 7(20), 35-52. Doi: 10.22201/enes1.20078064e.2019.20.68425

Steinwender, C. (2018). Real Effects of Information Frictions: "When the States and the Kingdom became United". American Economic Review, 108(3), 657-696. Doi: $10.1257 /$ aer.20150681

Tello, M. (2017). Cadenas globales de valor y exportaciones de productos intensivos en recursos naturales de América Latina y el Caribe: 1994-2011. Banco Interamericano de Desarrollo (BID). Nota Técnica, (IDB-TN-1241). Doi: https://doi. org/10.18235/0000614

Tyson, L. \& Lund, S. (2017). Globalization is not in Retreat: Digital Technology and the Future of Trade. Foreign Affairs, 97(3), 130-141. Doi: 10.1016/j. chieco.2017.08.010 